\title{
First bacteremia described by Butyricimonas virosa in Spain
}

\author{
Asunción Gasós ${ }^{1}$, Patricia Sigüenza ${ }^{2}$, Helena Monzón $^{2}$ and Emma Padilla ${ }^{1 *}$ \\ ${ }^{1}$ Microbiology Laboratory, CATLAB, Vial Sant Jordi s/n, Pol. Ind. Can Mitjans, 08232 Viladecavalls, Spain \\ ${ }^{2}$ Internal Medicine Unit, Fundació Hospital Sant Joan de Déu de Martorell, C/ Mancomunitats Comarcals 1-3, 08760 Martorell, Spain
}

\begin{abstract}
In the present document, we describe the case of bacteremia caused by Butyricimonas virosa (B. virosa), the first reported in Spain, occurred in a patient affected by colon adenocarcinoma. The literature is reviewed and we summarize the characteristics of the previously described cases, pointing out the fact that $B$. viros $a$ is closely related to intra-abdominal pathology, especially with gastrointestinal malignancy.
\end{abstract}

\section{Introduction}

Butyricimonas.spp.is a member of the family "Porphyromonadaceae", a group of bacteria first time isolated from rat faeces [1]. Two species have been described: Butyricimonas synergistica and Butyricimonas virosa [1]. Cells are Gram-negative, obligate anaerobic, non-pigmented, non-spore-forming and non-motile. The growth of the strains is inhibited in medium containing $20 \%$ bile. Colonies are opalescent, slightly convex and smooth in sheep agar, and the major end products are butyric and isobutyric acids [1]. B. virosa species exhibits additional characteristics: it produces a catalase, and gelatin is not digested in the cultures. Besides, B. virosa is the only pathogenic species within the recently described genus Butyricimonas [2], and has been associated with digestive pathology [2-5].

\section{Case Report}

A 72-year-old man was admitted to the emergency room (ER) at Sant Joan de Déu de Martorell Hospital (HSJDM), symptoms were: left hypochondrium pain, nausea, vomiting, and fever shaking chills. At physical examination, an erythemic left-hemiabdomen mass was described, and subcutaneous crepitation was also found in the described location. A blood test proved microcytic anemia, leukocytosis of 18.000/ $\mathrm{mm} 3$, with neutrophilia predominance, C-reactive protein at $382 \mathrm{mg} / \mathrm{L}$, lactacidemia of $6 \mathrm{mmol} / \mathrm{L}$, and altered coagulation test. An emergent CT scan was performed, showing a big mass in splenic flexure and abdominal wall, along with intestinal fistula complicated by cellulitis. Given the grievous condition of the patient, an empiric antibiotherapy with meropenem was indicated. Blood sampling specimens for cultures where previously taken. Emergency surgery was performed, consisting of hemicolectomy with terminal colostomy and drainage of abdominal cellulitis. The anatomopathological sample of the surgical piece offered a late diagnosis of colon adenocarcinoma, with lymph node involvement

The anaerobic blood specimen culture was positive on the fifth day of incubation for Gram-negative short bacilli, identified by direct Gram staining. Identification of the anaerobic Gram-negative bacteria through VITEK 2 ANC ID (BioMérieux) was not possibly possible. The isolated bacteria were then, evaluated by matrix-assisted laser disruption/ionization time-of-flight mass spectrometry (MALDI-TOF) Vitek MS (BioMérieux). Again, a conclusive identification could not be made. At last, the organism was identified as B. virosa by $16 \mathrm{~S}$ rRNA gene sequence analysis. Antibiotic susceptibilities were determined by E-test (bioMérieux), it showed resistance to penicillin G. On the other hand, sensitivity to amoxicillin-clavulanic acid, piperacillin-tazobactam, meropenem, clindamycin, and metronidazole was described.

On the basis of a clinical worsening of the patient, he was admitted to the Intensive Care Unit (ICU) presenting multiple complications, even requiring new surgery involving total colectomy. Respiratory and hemodynamic failure was observed, secondary to severe bleeding due to diffuse esophageal ischemia. In spite of having administered the right antibiotherapy, based on the E-test, the surgical and ICU resuscitation procedures the patient's clinical condition showed relative improvement just before a severe deterioration was observed, and died 3 weeks after admittance.

Intraabdominal infections are well-known sources of polymicrobial bacteremia. Anaerobes such as Bacteroides spp. and Clostridium spp account for 30-50\% of those cases [5]. Anaerobic bacteremia, accounting for $4 \%$ of all bacteremia cases at the HSJDM, are mostly originated from endogenous microbiome, often associated with a source of abdominal infection [2], especially in patients with malignancies (particularly colonic cancer). Nevertheless, a significant proportion of anaerobes remain unidentified. Butyricimonas virosa is a prevalent inhabitant of the intestine $[1,5]$. Few cases of human cases of infection due to $B$. virosa have been reported in literature [2-6], all of which were bacteremia cases, and three of them were associated with gastrointestinal tumors. On the case we report, the patient had an advanced colon malignancy (see Table). Precocious diagnosis is critical in those cases. Rapidly advancing technology, such as MALDI-TOF and 16S rRNA gene sequence, is now available to many clinical laboratories, and this advance has now led to the recognition of pathogens like B. virosa [6] and may lead to an improved patient handling, and to earlier, specific treatment for these

${ }^{\star}$ Correspondence to: Emma Padilla $\mathrm{PhD}, \mathrm{MD}$, Microbiolgy Laboratory, CATLAB, Vial Sant Jordi s/n, Pol. Ind. Can Mitjans, 08232 Viladecavalls, Spain, Tel: 34-937485600; Fax: 34-937485610; E-mail: epadilla@catlab.cat

Key words: Butyricimonas virosa, Bacteremia

Received: February 21, 2019; Accepted: February 26, 2019; Published: February 28,2019 
Table 1. Selected characteristics from cases of Butyricimonas bacteremia.

\begin{tabular}{|l|l|l|l|l|l|l|}
\hline & Age & Gender & Predisposing condition & Diagnostic method & Susceptibilities \\
\hline Toprak [4] & 72 & H & $\begin{array}{l}\text { Previous diagnosis colon } \\
\text { adenocarcinoma. Complication } \\
\text { after aneurysm surgery. }\end{array}$ & 16SrRNA & S \\
\hline Mehta [3] & 81 & H & $\begin{array}{l}\text { Duodenal adenocarcinoma } \\
\text { surgery. }\end{array}$ & MALDI-TOF & Reath kanamicin and colistin \\
\hline Enemchukwu [6] & 69 & H & $\begin{array}{l}\text { Divecovered without } \\
\text { antibiotic }\end{array}$ & Rolitis \\
\hline Ogawa [5] & 68 & H & $\begin{array}{l}\text { Colorectal surgery (gastric } \\
\text { cancer and appendiceal } \\
\text { carcinoma) }\end{array}$ & 16SrRNA & Surgery and recovered \\
\hline García-Agudo [2] & 90 & H & Appendicitis & 16SrRNA & Renicillin and ceftriaxone \\
\hline Gasós & 72 & H & Colon adenocarcinoma & 16SrRNA & R penicillin \\
\hline
\end{tabular}

infections. In four of the cases described the B. virosa identifications were by 16 SrRNA sequencing and the other two cases by MALDI-TOF. Even though the identification of the strain throughout MALDI-TOF is described, we weren't able to get any identification, probably because our software was not capable of identifying it.

Previous data on antimicrobial resistance of $B$. virosa reveals that most of the strains were resistant to penicillin and susceptible to amoxicillin-clavulanic and it's by-products, and clindamycin, metronidazole, and meropenem, but one of the reports showed that their isolated strain was sensitive to penicillin and it's by-products, and clindamycin, and metronidazole, too (Table 1).

In conclusion, is our purpose to emphasize that, even that $B$. virosa infections are infrequent, when detected they appear to be related to abdominal pathology and most cases to malignancy at such location. This is the first case of bacteremia caused by $B$. virosa described in our country, since precedents in the reviewed literature were not found, and is the fourth case reported, described along with significant clinical similarities with the rest of the cases. Further, molecular identifications technologies such as MALDI-TOF and 16SrRNA gene sequence provide microbiologists understanding of clinical syndromes and new pathogens.

\section{Transparency Declaration}

Conflict of interest disclosure: The authors declare they have no conflict of interest.
Funding: This research did not receive any specific grant from funding agencies in the public, commercial or not-for-profit sectors.

Author's contribution: EP, AG, PS and HM were involved in data acquisition. EP and PS were involved in data analysis and interpretation. EP drafted the manuscript. EP, AG, PS and HM revised the manuscript critically. All authors approved the final version to be submitted.

\section{References}

1. Sakamoto M, Takagaki A, Matsumoto K, Kato Y,Goto K, Benno Y (2009) Butyricimonas synergistica gen. nov., sp. nov.and Butyricimonas virosa sp. nov. butyricacid-producing bacteria in the family 'Porphyromonadaceae' isolated from rat faeces. Int J Syst Evol Microbiol 59: 1748-1753.

2. García-Agudo L, Nilsen E (2018) Butyricimonas virosa: A rare cause of bacteremia Anaerobe 54: 121-123. [Crossref]

3. Mehta SR, Estrada J, Basallo C, Farala A, Fierer J (2015) Butyricimonas virosa bacteraemia identified by MALDI-TOF. New Microbes New Infect 8: 127. [Crossref]

4. Ulger Toprak N, Bozan T, Birkan Y, Isbir S, Soyletir G (2015) Butyricimonas virosa: the first clinical case of bacteraemia. New Microbes New Infect 4: 7-8. [Crossref]

5. Ogawa Y, Sato M, Yamashita T, Nakano R, Mochizuki S, et al. (2018) Polymicrobial anaerobi bacteremia caused by.Butyricimonas virosa and Brachyspira pilosicoli in a patient with peritonitis folowing intestinal perforation. Ann Lab Med 38: 71-73.

6. 6. Enemchukwu CU, Ben-Faras H, Gialanella P, Szymczak WA, Nosanchuck JD, Madaline TF (2016). Butyricimonas virosa bacteremia and bowel disease: case report and review. New Microbes New Infect 13: 34-36.

Copyright: (C2019 Gasós A. This is an open-access article distributed under the terms of the Creative Commons Attribution License, which permits unrestricted use, distribution, and reproduction in any medium, provided the original author and source are credited. 